\title{
Facial basal cell carcinoma: Analysis of recurrence and follow-up strategies
}

\author{
LEONARDO SARTORE $^{1}$, LUCA LANCEROTTO ${ }^{1}$, MICHELA SALMASO ${ }^{1}$, \\ GIORGIO GIATSIDIS $^{1}$, OMAR PACCAGNELLA ${ }^{2}$, MAURO ALAIBAC ${ }^{3}$ and FRANCO BASSETTO ${ }^{1}$
}

${ }^{1}$ Unit of Plastic Surgery, ${ }^{2}$ Department of Statistical Sciences, and ${ }^{3}$ Unit of Dermatology, University of Padua, Padua, Italy

Received May 26, 2011; Accepted June 28, 2011

DOI: $10.3892 / o r .2011 .1453$

\begin{abstract}
Rates of recurrence after incomplete surgical excision of basal cell carcinoma (BCC) range from 4 to $16.6 \%$ of analyzed cases. The aim of the present study was to identify the predictive factors associated with facial BBC recurrence following excision and their influences, in order to establish a proper therapeutic strategy. A monocentric retrospective study was performed reviewing all BCCs surgically excised at the Institute of Plastic Surgery, University of Padua, with particular focus on the involvement of surgical margins and recurrence. Seven hundred and nineteen lesions in 605 patients were studied. Correlations between recurrence probability and various characteristics of BCC were analyzed using a logistic regression model. It was observed that incomplete excision, deep margin involvement, the presence of sclerodermiform or metatypic basaloid squamous cells, as well as pleomorphous histological variants and/or peritumoral inflammatory infiltrates, were all related to an increase in the probability of recurrence. BCC excision must be followed by individualized management with particular consideration for the localization, the histological type and other known predisposing factors; the treatment strategy and, in particular, the length of the surveillance period and the frequency of patient assessment should be evaluated on the basis of the recurrence probability outlined.
\end{abstract}

\section{Introduction}

According to current epidemiology, basal cell carcinoma (BCC) is the most common type of facial skin cancer. It represents alone approximately $65 \%$ of all epitheliomas (1), and the incidence is 4-fold higher than that of squamous cell carcinoma (2). Among predisposing factors, chronic exposure to the sun and/or to other types of ionising radiation plays a main role, particularly related to individual skin type, while other significant factors include immunosuppression, pre-existing cutaneous lesions and genetic conditions (e.g. albinism,

Correspondence to: Dr Mauro Alaibac, Unit of Dermatology, University of Padua, Via Giustiniani 2, 35128 Padua, Italy

E-mail: mauro.alaibac@unipd.it

Key words: basal cell carcinoma, skin cancer, treatment strategy xeroderma pigmentosum, and Gorlin-Goltz syndrome). The reported annual incidence is approximately 65/100,000 individuals in Europe, 146/100,000 in the US (3) and reaches $726 / 100,000$ in Australia (4). A higher frequency is noted in males (5) and in older age, with a peak around the age of 70 (6). Nearly $80-85 \%$ of BCCs involve the head and neck regions and $25-30 \%$ are localized in the nasal area. Other frequently involved anatomical regions are the cheeks, the retroauricular area, the forehead, the periorbital zone, and, in particular, the inner canthus (3). Although it is described as a slow growing tumour and metastases are extremely rare $(<0.1 \%)$, BCC may lead locally to considerable morbidity and complications, with particular reference to cases of recurrence following surgical excisions of primary lesions; these cases tend to be biologically more aggressive.

According to the literature, surgically excised primary BCCs may show a recurrence rate that varies from 5 to $14 \%$ (7-12). The rates of recurrence appear to depend, to a large extent, on the anatomical localization, the histological characteristics, the initial treatment strategy and the eventual neoplastic margin involvement. A variety of several therapeutic options have been proposed for the treatment of these lesions. Among these, surgical excision, Mohs micrographic surgery, curettage, diathermocoagulation (DTC), cryotherapy, photodynamic therapy, radiotherapy and application of local cytotoxic drugs have been used. Nevertheless, according to most eminent authors, surgery should be considered the gold standard therapeutical choice, particularly when histological tumour surveillance is guaranteed. As stated, lesions localized in the head and neck region are more at risk for recurrence with respect to those of the trunk or limbs (13). Moreover, various atypical histological characteristics are reported to be predictive of a significant increase in the recurrence rates such as a sclerodermiforme growth pattern, a squamous differentiation, an absence of or no clearly delimited peripheral palisading and a marked nuclear pleomorphism $(12,14,15)$. Moreover, recurrence rates reported for primary BCCs 5 years after complete excision account for $99 \%$ of cases of Mohs microsurgery, about $95 \%$ for traditional surgical excision, radiotherapy or cryosurgery and almost $92 \%$ with DTC (16). Conversely, the recurrence rate for incompletely excised BCC varies from 10 to $67 \%(12,13,17-27)$. Notwithstanding, the great disparity and variability in recurrence rates reported by different authors have contributed to the lack of an accepted 
agreement concerning the optimal therapeutic strategy to adopt in those cases in which histological examination shows an infiltration of surgical margins or a suboptimal margin free from disease. At our institution we reviewed the records of facial BCC surgical excisions during a 6-year period from January 2003 to December 2008 to evaluate the recurrence rates following complete or incomplete excision in the case of a prolonged follow-up approach vs. the case of immediate re-excision, considering characteristics of the patients and neoplasms likely influencing the probability of recurrence.

\section{Materials and methods}

A retrospective monocentric study was performed on the analysis of data pertaining to patients admitted at our institution for surgical excision of facial BCCs from January 2003 to December 2008. The experimental protocol of the study was designed according to the ethical guidelines of the Declaration of Helsinki of 1975, as revised in 1983. An informed consent was obtained from each of the 605 patients corresponding to a total of 719 primary excisions of facial neoplasms for which diagnosis of BBC was confirmed upon histological analysis. Data relative to gender, age, date of surgery, the existence of predisposing factors of either a genetic (in particular xeroderma pigmentosa, albinism, and basal cell nevomatosis) or an environmental (chronic sun exposition, facial exposure to radiation, immunosuppression) nature, the presence of other facial BCCs and the total number of BBCs excised over the 6-year study period, were all collected and digitally stored. Additionally collected clinical/histological data included the facial area involved, the histological characteristics of the lesion (histological type, presence of ulceration, degree of tissue involvement, the presence of peritumoral inflammatory infiltration) and surgical approach; whether re-excision was carried out immediately or a wait-and-look approach was preferred in cases of incomplete/suboptimal excision.

On the basis of histological reports, margins were classified into 5 groups according to whether the margins appeared to have no neoplastic involvement ('safe margin' $\geq 5 \mathrm{~mm}$ ) (Group one), or showed an involvement of one or more lateral margins (Group two), involvement of a deep margin (Group three), involvement of a deep margin together with at least one lateral margin (Group four), or suboptimal excision (margins not involved by disease but inadequate safe distance) (Group five).

Following resection, the patients were stringently monitored and examined at our outpatient clinic at least once a year. The follow-up period lasted from a minimum of 3 years to a maximum of 9 years. The histological features of the excised recurrent lesions were analyzed as well and compared with those of the primary lesions. Finally, presentation of new facial BCCs was also evaluated during follow-up examinations.

Concerning the statistical analysis, a preliminary analysis included elaboration of extensive descriptive statistics. Excised BCCs were divided into two groups depending on the presence or absence of recurrence. Contingency tables showing the distribution of each tumour characteristic in the two groups were developed. A p-value and Pearson's contingency coefficient were calculated for every table. Associations between the two variables being examined within these tables were evalu-

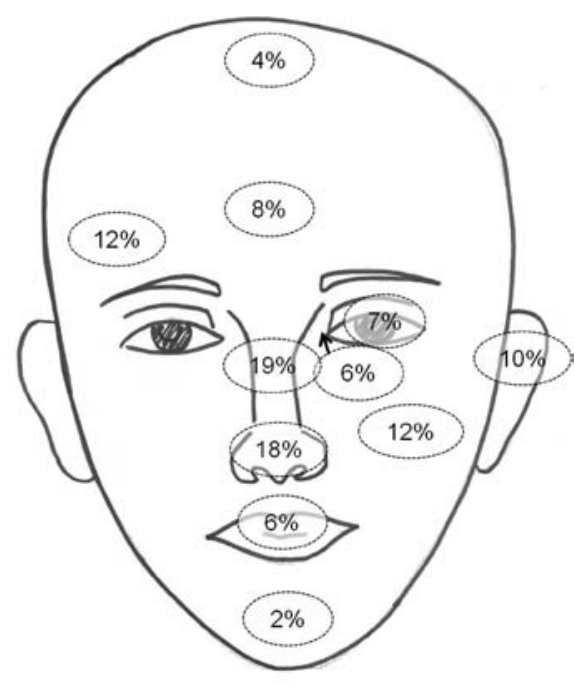

Figure 1. Anatomical distribution of the excised primary BCCs.

ated assuming positive or negative values (negative when the two variables were independent from one another and positive when there appeared to be an association).

\section{Results}

In total, 719 lesions in 605 patients [356 (58.8\%) males, and $249(41.2 \%)$ females, age range $28-95$ years, average 70 years] were collected, analysed and reviewed. During the 6-year study period, approximately 526 patients were treated for one $\mathrm{BCC}$, and multiple lesions were excised in $16 \%$ of the enrolled males and in $10.4 \%$ of the enrolled females ( $<<0.05$ ). Among these, 68 patients presented two either synchronous or metachronous BCCs while the remaining 19 patients presented with three each. Significant predisposing factors were noted in $10.6 \%$ of the lesions. In addition, nearly $18.8 \%$ of the lesions occurred in patients with a positive clinical history for facial BCCs. The anatomical distribution of the primary lesions is shown in Fig. 1.

Surgical management of the lesions included repair by direct suture in $45.5 \%$ of the excised lesions, repair by a skin graft in $29.5 \%$ of the cases and repair by local flap in the remaining $25 \%$. Reports of histological analysis showed that $77.1 \%$ of the examined primary BCCs were of nodular type, $21.7 \%$ had a sclerodermiforme morphology, $12.4 \%$ an adenoid, $11.1 \%$ a superficial conformation, $10.6 \%$ included a pilar differentiation and $3.7 \%$ had a metatypic-pleomorphicbasosquamous type. Ulceration occurred in $52.7 \%$ of the lesions, while in $2.5 \%$ of the cases an infiltration of the papillary dermis was noted. In $\sim 72.0 \%$ of the lesions, infiltration involved the reticular dermis, in $13.1 \%$ infiltration affected the hypodermis and in another $8.9 \%$ infiltration reached the deep structures (such as the muscular and bony structures). Inflammatory infiltrate was positive in $3.8 \%$ of the neoplasms. Surgical oncological outcome indicated that $550(75.6 \%)$ of the primary excisions performed were complete as determined by histological examination while 47 (6.5\%) were suboptimal. The remaining lesions $(122 / 16.9 \%)$ had partial infiltration of the margins; of these, $\sim 11.1 \%$ had involvement of the lateral 


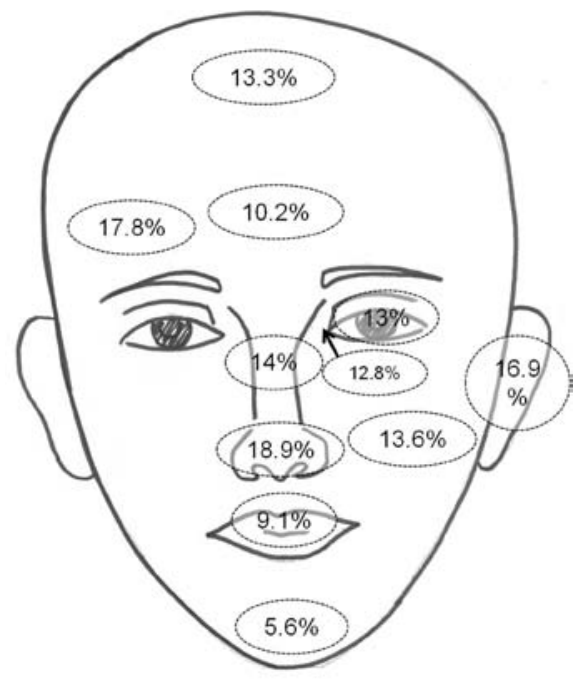

Figure 2. Recurrence rates of the excised BCCs depending on anatomical location.

margins, deep margins were affected in $3.1 \%$, and both lateral and deep infiltrations were noted in the remaining $2.8 \%$.

Recurrence rates. Considering a total of 719 excised primary lesions, the recurrence rate was assessed and recorded during follow-up in 105 cases, giving a global recurrence rate of $14.6 \%$.

Anatomical distribution. The lesions located on the nasal ala showed the highest recurrence rate $(18.9 \%)$, while those on the lip (9.1\%) and the chin (5.6\%) showed the lowest percentages; nevertheless, differences were not significant (Fig. 2).

Patient history. Recurrence of BCCs was noted in $26.3 \%$ of the patients with predisposing factors (either genetic or environmental), compared to $13.3 \%$ in patients without predisposing factors $(p<0.01)$. Moreover, in subjects with a history of previous facial BCCs, recurrence occurred in $23 \%$ of the cases $(\mathrm{p}<0.01)$ vs. $12.7 \%$ in patients without a known positive history.

Excision and repair. We observed that BCCs with optimal excision had a recurrence rate of $8.4 \%$. In contrast, when the margin involvement was assessed (lateral, deep, or both) recurrence was $38.5 \%$. Finally, lesions with suboptimal excision had a recurrence rate of $25.5 \%(\mathrm{p}<0.01)$. No other significant differences in recurrence rates were found for either gender or age. Considering the surgical outcome according to the chosen surgical repair technique, $17.9 \%$ of the lesions repaired with skin grafts showed recurrence compared to $14.4 \%$ when direct suture repair was performed and $11.1 \%$ when a flap was used to repair the lesion.

Histology. BCCs presenting infiltration limited to the dermis papillary had a recurrence rate of $5.6 \%$, and those with infiltration of the dermis reticular exhibited a $13.5 \%$ recurrence. In cases in which extension included the hypodermis, the recurrence rate reached $13.8 \%$, and the rate increased to $21.9 \%$ when the tumour extended to underlying structures.
According to histological type, the highest recurrence rates were noted in the metatypic-basaloid squamous-pleomorphous $(29.6 \%)$ and the sclerodermiforme $(25.7 \%)$ variants. In contrast, lower percentages were noted in BCCs with pilar $(15.8 \%)$, adenoid $(13.5 \%)$, nodular $(12.5 \%)$ and superficial $(11.3 \%)$ differentiation characteristics. Finally, $48.2 \%$ of BCCs with an inflammatory peritumoral infiltration exhibited recurrence. We observed that differences in the histological pattern of the primary lesion with respect to the recurrent lesion were quite frequent $(57.1 \%)$. Sclerodermiforme characteristics that were almost absent in the primary lesion were evident in $37.9 \%$ of recurrences. Moreover, while almost $77 \%$ of the original 713 lesions presented nodular characteristics only $53.3 \%$ showed the same features in the recurrent lesions. Sclerodermiforme characteristics were found in $21 \%$ of the original lesions and were expressed in $50.5 \%$ of the recurrent ones. Again, the superficial type was observed in only $4.8 \%$ of cases while $>21 \%$ of the recurrences presented other characteristics (adenoid, pilar and metatypic-basaloid squamous cell-pleomorphous features).

Analysis of the probability of recurrence. The calculated recurrence probability was $\sim 16$-fold greater for lesions with positivity for two margins when compared to lesions with complete excision. The recurrence probability was, likewise, 9.5-, 9.0- and 4.7-fold greater, respectively, for lesions with positive deep margins, positive lateral margins and suboptimal excision when compared to lesions completely excised $(\mathrm{p}<0.01)$. Gender and age were not significantly correlated with recurrence.

In addition, the recurrence probability was 2.7 higher $(\mathrm{p}<0.01)$ for patients with determined predisposing factors. A similar trend was also noted for variables regarding a precedent facial BCC, even when the odds ratio was not statistically significant. After evaluation of the histological characteristics of the BCCs, sclerodermiforme and the metatypic-basaloid squamous cell-pleomorphous patterns were significantly predisposed to recurrence $(\mathrm{p}<0.01)$, and the recurrence probability increased, respectively, 2.5 -and 4.5 -fold with respect to other types of BCCs. Depth of infiltration did not correlate with statistical significant variations in recurrence probability.

Recurrence rates for lesions repaired with skin graft or direct suture were similar. Cases in which the excision was repaired with a local skin flap, instead, presented a lower recurrence rate: 0.5 -fold decrease with respect to a direct suture repair $(\mathrm{p}<0.1)$. All of the anatomical sites specified in the model presented an odds ratio less than one. According to this finding, the recurrence probability tended to be lower when the tumour was located in an area other than the nasal ala, while primary location to the lips or root of the nose significantly correlated with a higher probability of recurrence $(\mathrm{p}<0.01$ and $\mathrm{p}<0.05$, respectively).

Analysis of recurrence timing (Figs. 3 and 4). Approximately $45.7 \%$ of recurrences occurred within 12 months of excision, while totally $61 \%$ were evident within 24 months and $77.1 \%$ within a 3 -year time span. Approximately $1 / 4$ of all recurrences had a clinical appearance within 6 months. When excised primary lesions showed infiltration of margins, $>50 \%$ of recurrences were observed within one year of excision; a 


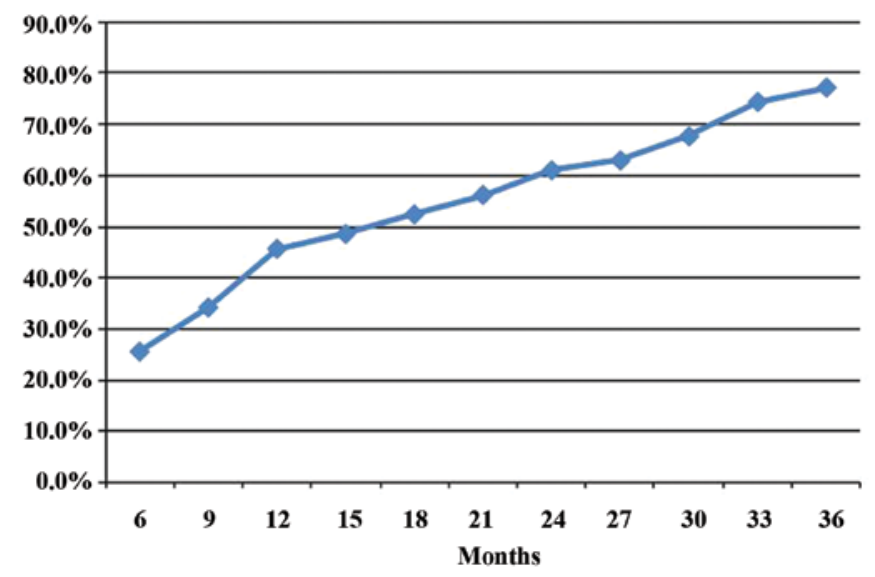

Figure 3. Timing of recurrence over the 36 months following primary BBC excision. Percentages relate to the total number of recurrent BCCs.

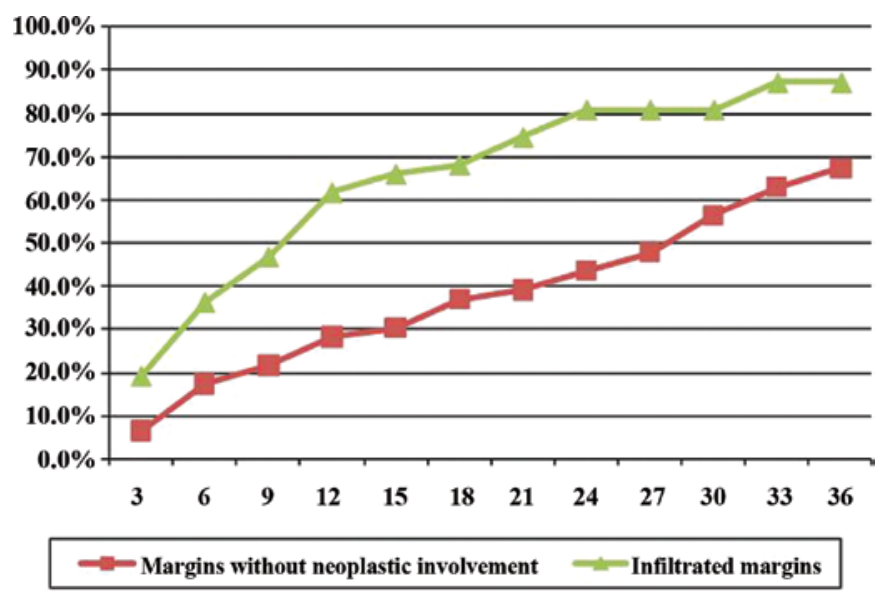

Figure 4. Timing of recurrence depending on the absence/presence of margin infiltration at excision of the primary lesion. Percentages of the total number of recurrent BCCs.

percentage that reached $90 \%$ within 3 years of surgery. In contrast, recurrence of lesions that were adequately excised developed with longer latency; $50 \%$ of recurrences in these cases were reported after 27 months. BCCs with completely excised margins had the same recurrence rate during the first year after excision. However, during the ensuing years, recurrence showed a linear pattern in the course of the 36 months studied.

\section{Discussion}

The epidemiologic characteristics of our patient group were substantially similar to other reports published in the literature. Overall, neoplastic margin involvement at histological examination was present in 17\% (122 lesions) of excisions in our cases, with a $6.5-20.7 \%$ range as reported in the literature (23). The global recurrence rate assessed (14.7\%) was within the $5-19 \%$ range reported in most previous studies $(7-9,11,12,27)$. Regarding surgical excision, a $38.5 \%$ recurrence was noted when histological analysis showed an infiltration (lateral, deep or both margins infiltration), while a $25.5 \%$ recurrence rate for lesions with suboptimal excision can be expected and a $8.4 \%$ recurrence with histologically confirmed optimal excision, nevertheless, was recorded during the follow-up. According to some investigators this fact may be explained considering that standard histological procedures may lead to an inaccurate analysis of a percentage of tissue samples $(26,28)$. Moreover, a further explanation may be found in the predisposition of the individual patient to develop skin neoplasms, and the recurrence may actually be the macroscopic clinical manifestation of a newly developed $\mathrm{BCC}$ or a lesion already present nearby the primary lesion. For these reasons, periodic follow-up even for patients with completely excised lesions appears, in any case, advisable. The frequent absence of any recurrence in cases in which histological examination diagnosed an infiltration of any margin still requires explaination. In the present study this situation occurred in $52(65 \%)$ out of 80 BCCs with positive lateral margins and in $23(55 \%)$ of the lesions presenting infiltration of a deep margin. Most previous studies have reported similar observations, and many research groups agree that the peak time of recurrence occurs during the second year following excision $(13,17,29)$. The followup of patients in our study was for a minimum of 3 years, a likely sufficient time period during which to observe clinical manifestations of recurrence according to the literature. In addition, a spontaneous regression of basal cells may take place as a result of an immunologic reaction. Indeed, Dellon et al observed a direct correlation between regression and the consistency of lymphocyte infiltration (12). On the other hand, a weak immune response to the host cells may increase the risk of recurrence. Other authors have suggested that surgery and the consequent scarring reaction may cause a devitalisation of residual tumoral cells (30). In our experience, consistent with other studies (31), factors in the patient history having a statistically significant influence $(\mathrm{p}<0.05)$ on recurrence were history of facial BCCs and the presence of predisposing factors (either genetic or environmental). Furthermore, among histological variables, the sclerodermiforme feature was associated with a $25.7 \%$ recurrence rate and the highest percentage of infiltrated margins (23\%). This finding may be explained by the fact that in this histological type of tumour the margins are clinically difficult to identify and the actual extension of the lesion may be underestimated at the time of excision (1). The metatypicbasaloid squamous cell and pleomorphous histological variants exhibited a $30 \%$ recurrence rate, and in $7.4 \%$ of the cases their margins were infiltrated. The metatypic basal cell histological variant has been the subject of debate regarding its histological definition (32); it appears more appropriate to define its features as falling somewhere between basal cell and spinocellular carcinomas, for which they share the same spreading rate. However, SCCs tend to be more aggressive presenting a greater incidence of lymphatic diffusion and peritumoral infiltration $(6,32)$. Basaloid squamous BCC is diagnosed accurately when areas of basal cell and squamous carcinomas are noted with a minimum area of mixture between the two $(6,32)$. Pleomorphous (basal cell carcinomas with monster cells) BCCs exhibit characteristics of the nodular basal cell type, while nuclear aneuploidy is considered a marker of histological aggressiveness (33). In our study, basal cell carcinomas with inflammatory infiltrates presented a recurrence rate of $48.2 \%$, a finding that is in disagreement with other reports. 
ONCOLOGY REPORTS 26: 1423-1429, 2011

1427

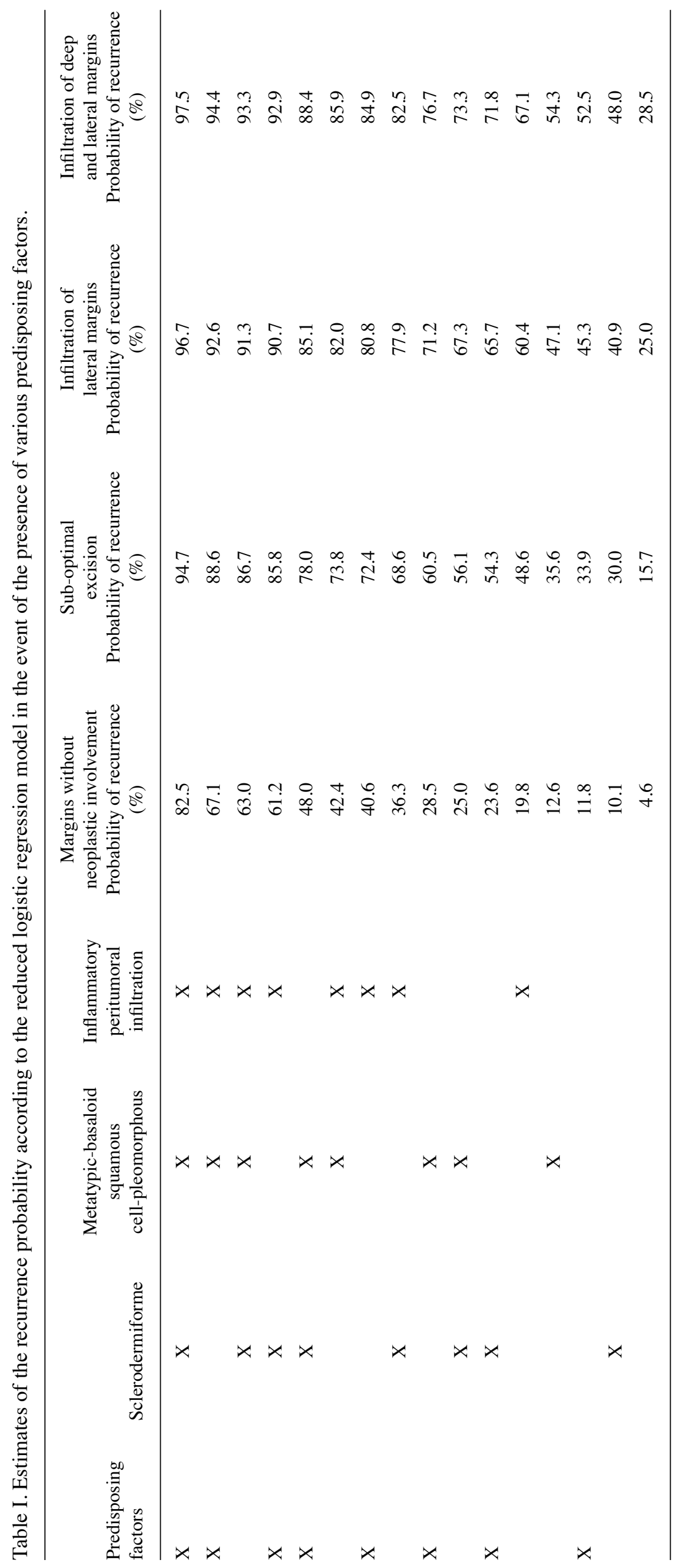


Dellon et al (12) suggested that peritumoral lymphocytic infiltration may decrease the risk of recurrence influencing the regression of residual neoplastic cells. In addition, it has been hypothesized that T CD4 ${ }^{+}$lymphocytes, generally preponderant within the peritumoral inflammatory infiltrate, may induce regression through cytokine production (34). Nonetheless, in our observations, the inflammatory infiltrates were assessed with standard pathology haematoxylin-eosin staining analysis which, unlike immunohistochemical stainings, does not permit cell typization. Thus, the presence of inflammatory cells different from T CD4 ${ }^{+}$lymphocytes may explain the discrepancy with data present in the literature and may play an important, yet unknown, role in the biology of this tumour. In particular, a recent study suggested that mast cells play an active role in promoting the angiogenesis of basal cell carcinomas by producing vascular endothelial growth factor (VEGF) and IL-8 (35). Moreover, our study pointed out the eventuality of a change-over in the histological pattern from the primary lesion to the recurrent lesion. Indeed, sclerodermiforme characteristics that were absent in the primary lesion were present in $37.9 \%$ of the recurrent lesions: this was a frequent finding (57.1\% of recurrences) and it is in accordance with other reports in the literature (18), although few studies on this subject have been published. These findings support the hypothesis that recurrent lesion are biologically different entities characterized by greater aggressiveness than primary lesions.

According to the multivariate analysis (logistic regression model), in particular, our study demonstrated the following. i) The probability of recurrence of BCC in subjects with predisposing factors was approximately 2.7 -fold higher with respect to that in subjects without. ii) Histologically diagnosed margin infiltration had an undeniable role in predisposing to recurrence. iii) Flap repair appeared to act as a preventive measure with respect to a direct suture repair for several likely explanations: excision margins may be wider when a local flap is adopted and the almost immediate flow of blood restoration may explain a greater inflammatory reaction capable of causing regression of residual basal cells. iv) The recurrence probability decreased when the lesion was localized in areas besides the nasal ala region, and the decrease was greater for sites such as the chin and the lips. This trend further confirms that BCCs developing along the embryological fusion planes delineating the $\mathrm{H}$ zone are at risk of recurrence independent of margin infiltration $(8,19,31)$. v) The recurrence probability for BCCs immediately re-excised decreased to approximately $85 \%$ with respect to tumours with positive margins that did not undergo surgical excision. Several studies have confirmed that immediate re-excision has a protective effect $(17,25)$.

On the basis of the estimates obtained with the predictive model, the recurrence probability for a patient can be calculated considering histological margin categories (complete excision, infiltration of the lateral margins, infiltration of the deep margins with or without lateral margins, suboptimal excision) and the presence of factors significantly correlated with recurrence, as outlined in Table I. This consideration may aid in the management of follow-up proposed to BCC patients.

In our model, the recurrence probability varied from a minimum of $4.6 \%$ to a maximum of $98 \%$. A probability level beyond which it is not opportune to pursue a wait-and-see approach cannot be established a priori. This means that, independently of the recurrence probability, other individual factors should be taken into account by the surgeon when considering whether or not to proceed further with surgery. We propose an immediate excision enlargement when the recurrence probability is higher than $25 \%$. It should be noted that neoplastic margin infiltrations always lead to a recurrence probability greater than $25 \%$ and that the presence of negative prognostic factors naturally increases this probability. Therefore, our proposed model may be helpful in dubious cases of suboptimal excision or optimal excision in the presence of various predisposing factors. When considering suboptimal excision, the recurrence probability varies from a minimum of $15.7 \%$ (absence of all negative prognostic factors) to a maximum of $97 \%$ (presence of all negative factors), while regarding completely excised tumours the probability range varies from a minimum of $4.6 \%$ to a maximum of $89.3 \%$. Therefore, in the latter condition, as well as for optimal excision, patients should be advised to undergo surveillance when the calculated risk for recurrence is greater than $25 \%$.

Some authors assert that an appropriate follow-up should not last less than 5 years (16), while others advise a 10 -year period after which the risk of recurrence of BCC with infiltrated margins is equal to that of a completely excised lesion (29). In our experience, the timeline of recurrence we observed suggests that a follow-up period of at least 3 years is adequate. Consistent with reports from other authors $(13,17,18)$, this management should allow for the early detection of recurrence and ensure more conservative treatment strategies at least in 3/4 of the cases. When the margins were found to be infiltrated, the recurrence rate reached more than $50 \%$ within 12 months from excision and $90 \%$ within 36 months. Considering this trend (Fig 4), a different follow-up schedule for the first year with respect to subsequent years seems advisable. Our protocol for patients with infiltrated/suboptimal margins ensures assessment at an outpatient clinic every 3 months for the first 12 months from surgery and every 6 months afterwards. On the other hand, recurrence of adequately excised lesions occurred slower and with a linear trend in the 36 months analyzed, therefore, annual examinations in an outpatient clinic may thus be scheduled for these patients.

\section{References}

1. Burg G, Hirsch RD, Konz B and Braun-Falco O: Histographic surgery: accurancy of visual assessment of the margins of basal cell epithelioma. J Dermatol Surg 1: 21-24, 1975.

2. Andrade R, Gumport SL, Popkin G and Reese R: Cancer of the skin. WB Saunders, Philadelphia, PA, 1976.

3. Miller DL and Weinstock MA: Non-melanoma skin cancer in the United States: incidence. J Am Acad Dermatol 30: 774-778, 1994.

4. Marks R, Staples M and Giles GC: Trends in non-melanocytic skin cancer treated in Australia: the second national survey. Int $\mathrm{J}$ Cancer 53: 585-590, 1993.

5. Czarnecki D, Meehan C, O'Brien T, et al: The changing face of skin cancer in Australia. Int J Dermatol 30: 715-717, 1991.

6. Goldberg LH: Basal cell carcinoma: Lancet 347: 663-667, 1996.

7. Panje WR and Ceilley RI: The influence of embryology of the midface on the spread of epithelial malignancies. Laryngoscope 89: 1914-1920, 1979.

8. Menn H, Robins P, Knopf AW and Barts RS: The recurrent basal cell epithelioma: a study of 100 cases of recurrent retreated basal cell epithelioma. Arch Dermatol 103: 628-631, 1971. 
9. Levine HL and Bailin PL: Basal cell carcinoma of the head and neck: identification of the high risk patient. Laryngoscope 90: 955-961, 1980.

10. Koplin L and Zarem HA: Recurrent basal cell carcinoma. A review concerning the incidence, behavior, and management of recurrent basal cell carcinoma, with emphasis on the incompletely excised lesion. Plast Reconstr Surg 65: 656-664, 1980.

11. Dubin N and Kopf AW: Multivariate risk score for recurrence of cutaneous basal cell carcinomas. Arch Dermatol 119: 373-377, 1983.

12. Dellon AL, De Silva S, Connoly M and Ross A: Prediction of recurrence in incompletely excised basal cell carcinoma. Plast Reconstr Surg 75: 860-871, 1985.

13. Sussman LA and Liggins DF: Incompletely excised basal cell carcinoma: a management dilemma? Aust NZ J Surg 66: 276-278, 1996.

14. Lang PG and Maize JC: Histologic evolution of recurrent basal cell carcinoma and treatment implications. J Am Acad Dermatol 14: 186-196, 1986.

15. Dixon AY, Lee SH and McGregor DH: Factors predictive of recurrence of basal cell carcinoma. Am J Dermatopathol 11: 222-232, 1989

16. Rowe DE, Carroll RJ and Day CL Jr: Long-term recurrence rates in previously untreated basal cell carcinoma: implications for patient follow-up. J Dermatol Surg Oncol 15: 315-328, 1989.

17. Richmond JD and Davie RM: The significance of incomplete excision in patients with basal cell carcinoma. Br J Plast Surg 40 63-67, 1987.

18. Freeman RG and Duncan WC: Recurrent skin cancer. Arch Dermatol 107: 395-399, 1973.

19. Wentzell JM and Robinson JK: Embryologic fusion planes and the spread of cutaneous carcinoma: a review and reassessment. J Dermatol Surg Oncol 16: 1000-1006, 1990.

20. Sei JF: [Excision limits and reoperation in cutaneous carcinoma] Ann Dermatol Venereol 124: 421-426, 1997 (In French).

21. Rowe DE, Carrol RJ and Day CL Jr: Mohs surgery is the treatment of choice for recurrent basal cell carcinoma. J Dermatol Surg Oncol 15: 424-431, 1989.

22. Silvermann MK, Kopf AW and Grin CM: Recurrence rates of treated basal cell carcinoma. Part 3: surgical excision. J Dermatol Surg Oncol 18: 471-476, 1992.
23. Hauben DJ, Zirchin H, Mahler D and Sacks M: The biologic behaviour of basal cell carcinoma: analysis of reccurence in excised basal cell carcinoma. Part II. Plast Reconstr Surg 69: 110-116, 1982.

24. Friedmann HI, Williams T, Zamora S and al-Assaad ZA: Recurrent basal cell carcinoma in margin-positive tumors. Ann Plast Surg 38: 232-235, 1997.

25. De Silva SP and Dellon AL: Recurrence rate of positive margin of basal cell carcinoma: results of a five-year prospective study. J Surg Oncol 28: 72-74, 1985.

26. Biely HC and Kirsen RS: Use of Mohs for determination of residual tumour in incompletely excised basal cell carcinoma. J Am Acad Dermatol 23: 754-756, 1992.

27. Holmkvist KA, Rogers GS and Dahl PR: Incidence of residual basal cell carcinoma in patients who appear tumour free after biopsy. J Am Acad Dermatol 41: 600-605, 1999.

28. Seidman JD, Barman JJ and Moore GW: Basal cell carcinoma: importance of histologic discontinuities in the evaluation of resection margins. Mod Pathol 4: 325-330, 1991.

29. Pascal RR, Hobby LW, Latters R and Crikelair GF: Prognosis of 'incompletely excised' versus 'completely excised' basal cell carcinoma. Plast Reconstr 41: 328-332, 1968.

30. Satura CY and Calamel PM: Comparison of treatment modalities for recurrent basal cell carcinoma. Plast Reconstr Surg 63: 492-496, 1979.

31. Bompous JM, Padhya TA and Barnett SN: Basal cell carcinoma of the head and neck: identification of predictors of recurrence. Ear Nose Throat 79: 200-204, 2000.

32. de Faria JL: Basal cell carcinoma of the skin with areas of squamous cell carcinoma: a basosquamous cell carcinoma. J Clin Pathol 38: 1273-1277, 1985.

33. Garcia JA, Cohen PR, Herzberg AJ, et al: Pleomorphic basal cell carcinoma. J Am Acad Dematol 32: 740-746, 1995.

34. Hunt MJ, Halliday GM, Weedon D, et al: Regression in basal cell carcinoma: an immunohistochemical analysis. Br J Dermatol 130: 1-8, 1994.

35. Aoki M, Pawankar R, Yayoi N and Kawana S: Masts cells in basal cell carcinoma express VEGF, IL-8 and RANTES. Int Arch Allergy Immunol 130: 216-223, 2003. 